International Journal of Mathematical, Engineering and Management Sciences

Vol. 3, No. 2, 151-166, 2018

https://dx.doi.org/10.33889/IJMEMS.2018.3.2-012

\title{
Note on History of Age Replacement Policies
}

\author{
Toshio Nakagawa \\ Department of Business Administration \\ Aichi Institute of Technology, Toyota 470-0392, Japan \\ Mingchih Chen \\ Graduate Institute of Business Administration \\ Fu Jen Catholic University, New Taipei City 24205, Taiwan \\ Xufeng Zhao \\ College of Economics and Management \\ Nanjing University of Aeronautics and Astronautics, Nanjing 211106, China \\ Corresponding author: xz.cem@nuaa.edu.cn
}

(Received March 22, 2017; Accepted September 27, 2017)

\begin{abstract}
This paper tries to trace our research history briefly from Barlow and Proschan to attain general replacement models. We begin with a random age replacement policy that is planned at a random time $Y$ and call it as random replacement. When the distribution of $Y$ becomes a degenerate distribution placing unit mass at $T$, age replacement is formulated. We obtain the general formulas for optimum replacement times. We next suppose the unit works for a job with random works, and replacement policies with $N$ cycles are discussed. As follows, we combine age and random replacement models and discuss replacement first, replacement last, replacement overtime, replacement overtime first and replacement overtime last. By formulating the distributions of replacement times with $n$ variables, general replacement models with $n$ replacement times are obtained.
\end{abstract}

Keywords- Age replacement, Replacement first, Replacement last, Replacement overtime, General replacement.

\section{Introduction}

Most basic model in maintenance theory is age replacement, in which we plan to replace an operating unit before failure at an optimum time $T^{*}$ to minimize the expected replacement cost rate. Since Barlow and Proschan (Barlow and Proschan, 1996) have introduced age replacement model in 1965, a great number of age replacement models have been discussed by researchers for a half century, which have been summarized (Barlow, and Proschan, 1996; Nakagawa, 2005; Sarkar et al., 2011). Recently, we have proposed several new notions of age replacement such as random replacement, replacement first, replacement last, replacement overtime, and replacement middle (Nakagawa, 2014; Zhao and Nakagawa, 2012; Nakagawa and Zhao, 2015; Zhao et al., 2015). General replacement models combing age and random replacement policies with $n$ replacement times have been studied (Chen et al., 2016).

This paper has an object of tracing our research history briefly from Barlow and Proschan (Barlow and Proschan, 1996) to attain general replacement models, which is shown in Fig. 1. 
International Journal of Mathematical, Engineering and Management Sciences

Vol. 3, No. 2, 151-166, 2018

https://dx.doi.org/10.33889/IJMEMS.2018.3.2-012

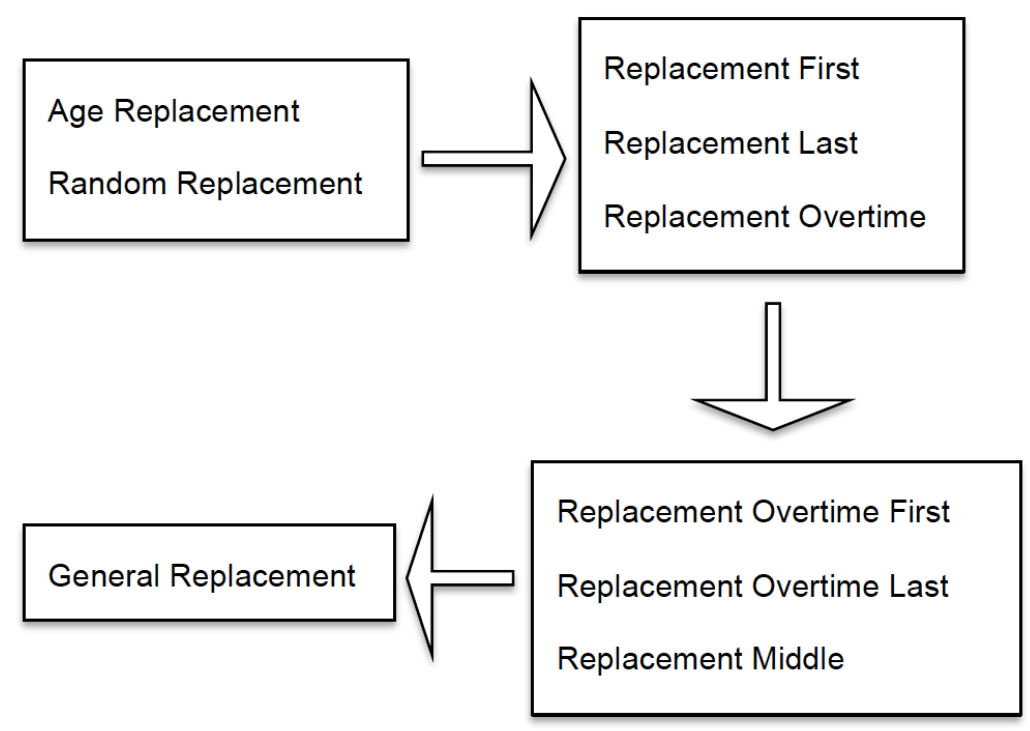

Fig. 1. The evolution of age replacement policies

In Section 2, general assumptions are given for modelings. In Section 3, we introduce age and random replacement models (Barlow and Proschan, 1996). In Sections 4-6, we give models of replacement first, replacement last, replacement overtime and replacement middle, respectively, and compare their optimum policies analytically. When the costs of preventive replacement are the same, age replacement is most economical than others. However, when a unit works for a job with random works, it is not wise in practice to stop its operation and to replace it at the optimum time, e.g., planned time $T$. In this situation, replacement overtime would be better than others. In Section 7, we propose general replacement policies and call them as redundant maintenances in general forms, as their distributions of replacement times agree with the failure distributions of a series system and a parallel system with $n$ units.

\section{General Assumptions}

We suppose that an operating unit has a failure distribution $F(t)$ with finite mean $\mu \equiv \int_{0}^{\infty}$ $\bar{F}(t) d t$, where $\bar{\varphi}(t) \equiv 1-\varphi(t)$ for any distribution $\varphi(t)$. Denote that $F(t)$ has a density function $f(t) \equiv \mathrm{d} F(t) / \mathrm{d} t$, i.e., $F(t)=\int_{0}^{t} f(u) d u$, and suppose the failure rate $h(t) \equiv$ $f(t) / \bar{F}(t)$ for $\bar{F}(t)<1$ increases strictly from $h(0) \equiv 0$ to $h(\infty) \equiv \lim _{t \rightarrow \infty} h(t)=\infty$.

In addition, we suppose the unit operates for an infinite time horizon by means of replacement. All times for corrective replacement and preventive replacement are supposed to be neglected and their costs are constantly given respectively. Our objective is to find optimum preventive replacement times to minimize the expected cost rates. 
International Journal of Mathematical, Engineering and Management Sciences

Vol. 3, No. 2, 151-166, 2018

https://dx.doi.org/10.33889/IJMEMS.2018.3.2-012

\section{Age and Random Replacement Models}

Suppose that an operating unit is replaced at a random time $Y(0<Y \leq \infty)$ or at failure, whichever occurs first. The random variable $Y$ has a general distribution $G(t)$ and is independent with the failure time $X$. We call this policy as random replacement. The expected cost rate is (Barlow and Proschan, 1996; Nakagawa, 2014)

$C(G)=\frac{c_{R}+\left(c_{F}-c_{R}\right) \int_{0}^{\infty} \bar{G}(t) d F(t)}{\int_{0}^{\infty} \bar{G}(t) \bar{F}(t) d t}$

where $c_{R}=$ replacement cost at time $Y, c_{F}=$ replacement cost at failure and $c_{F}>c_{R}$.

It has been shown (Barlow and Proschan, 1996; Nakagawa, 2014) that $C(G)$ can be rewritten as

where

$$
C(G)=\frac{\int_{0}^{\infty} Q(t) d G(t)}{\int_{0}^{\infty} S(t) d G(t)},
$$

$Q(t) \equiv c_{R}+\left(c_{F}-c_{R}\right) F(t)$ and $S(t) \equiv \int_{0}^{t} \bar{F}(u) d u$.

Suppose that there exits a minimum value $T(0<T \leq \infty)$ for $Q(t) / \mathrm{S}(t) \geq \mathrm{Q}(T) / \mathrm{S}(T)$, i.e.,

$$
\int_{0}^{\infty} Q(t) d G(t) \geq \frac{Q(T)}{S(T)} \int_{0}^{\infty} S(t) d G(t)
$$

Thus,

$$
C(G) \geq \frac{Q(T)}{S(T)} \geq C\left(G_{T}\right)
$$

where $G_{T}(t)$ is the degenerate distribution placing unit mass at $T$, i.e., $G_{T}(t) \equiv 1$ for $t \geq T$ and $G_{T}(t) \equiv 0$ for $t<T$. If $T=\infty$, then the unit is replaced at failure.

It was written in (Barlow and Proschan, 1996) that the elegant proof was due to S. Karlin, who was the author of (Karlin and Taylor, 1975). This is very easy and simple, however, it could not be occurred to anybody from (1). In other words, if preventive replacement costs are equal, the policy at time $T$ is superior to other ones, which will be shown theoretically in the following sections.

Suppose the unit is replaced at time $T$ or at failure, whichever occurs first. We call this policy as age replacement, and the expected cost rate is

$C(T)=\frac{c_{T}+\left(c_{F}-c_{T}\right) F(T)}{\int_{0}^{T} \bar{F}(t) d t}$,

where $c_{T}=$ replacement cost at time $T$ with $c_{T}<c_{F}$. Optimum $T^{*}$ to minimize $C(T)$ satisfies 
International Journal of Mathematical, Engineering and Management Sciences

Vol. 3, No. 2, 151-166, 2018

https://dx.doi.org/10.33889/IJMEMS.2018.3.2-012

or

$$
h(T) \int_{0}^{T} \bar{F}(t) d t-F(T)=\frac{c_{T}}{c_{F}-c_{T}}
$$

$\int_{0}^{T} \bar{F}(t)[h(T)-h(t)] d t=\frac{c_{T}}{c_{F}-c_{T}}$

which is the standard formula of deriving optimum age replacement time, and the resulting cost rate is

$C\left(T^{*}\right)=\left(c_{F}-c_{T}\right) h\left(T^{*}\right)$.

Equation (3) means physically that optimum replacement time satisfies: Failure rate $\times$ Mean replacement time - Failure probability $=$ Replacement cost ratio. This general formula will be shown in all sections.

Next, the unit works for a job with working cycles $Y_{j}(j=1,2 \cdots)$, where $Y_{j}$ are independent and have an identical distribution $G(t) \equiv \operatorname{Pr}\left\{Y_{j} \leq t\right\}$ with finite mean $1 / \theta \equiv \int_{0}^{\infty} \bar{G}(t) d t$. It is assumed that $G^{(j)}(t) \equiv \operatorname{Pr}\left\{Y_{1}+Y_{2}+\cdots+Y_{j} \leq t\right\}(j=1,2, \cdots)$ is the $j$-fold Stieltjes convolution of $G(t)$ with itself and $G^{(0)}(t) \equiv 1$ for $t \geq 0$.

Suppose that the unit is replaced at cycle $N$ or at failure, whichever occurs first. We also call this policy as random replacement, and the expected cost rate is (Nakagawa, 2014).

$C(N)=\frac{c_{N}+\left(c_{F}-c_{N}\right) \int_{0}^{\infty}\left[1-G^{(N)}(t)\right] d F(t)}{\int_{0}^{\infty}\left[1-G^{(N)}(t)\right] \bar{F}(t) d t}$

where $c_{N}=$ replacement cost at cycle $N$ with $c_{N}<c_{F}$. The expected cost rate $C(N)$ is easily obtained by replacing $G(t)$ and $c_{R}$ in (1) with $G^{(N)}(t)$ and $c_{N}$, respectively.

Optimum $N^{*}$ to minimize $C(N)$ satisfies

$\int_{0}^{\infty}\left[1-G^{(N)}(t)\right] \bar{F}(t)[Q(N)-h(t)] d t \geq \frac{c_{N}}{c_{F}-c_{N}}$,

where

$Q(T, N)=\frac{\int_{0}^{T}\left[G^{(N)}(t)-G^{(N+1)}(t)\right] d F(t)}{\int_{0}^{T}\left[G^{(N)}(t)-G^{(N+1)}(t)\right] \bar{F}(t) d t}<h(T)$,

and $Q(N) \equiv \lim _{T \rightarrow \infty} Q(T, N)$.

In particular, when $G(t)=1-e^{-\theta t}$, 
International Journal of Mathematical, Engineering and Management Sciences

Vol. 3, No. 2, 151-166, 2018

https://dx.doi.org/10.33889/IJMEMS.2018.3.2-012

$$
Q(T, N)=\frac{\int_{0}^{T}(\theta t)^{N} e^{-\theta t} d F(t)}{\int_{0}^{T}(\theta t)^{N} e^{-\theta t} \bar{F}(t) d t}
$$

increases strictly with $N$ to $h(T)$ and increases with $T$ to $Q(\infty, N)$ [4]. When $h(\infty)=\infty$ and $G(t)=1-e^{-\theta t}$, both optimum $T^{*}$ and $N^{*}$ to satisfy (3) and (6) exist.

\section{Replacement First and Last}

\subsection{Replacement First}

Suppose the unit is replaced preventively at $T$ or at cycle $N$, whichever occurs first. Then, the expected cost rate is (Nakagawa, 2014)

$C_{F}(T, N)=\frac{c_{T}+\left(c_{F}-c_{T}\right) \int_{0}^{T}\left[1-G^{(N)}(t)\right] d F(t)+\left(c_{N}-c_{T}\right) \int_{0}^{T} \bar{F}(t) d G^{(N)}(t)}{\int_{0}^{T}\left[1-G^{(N)}(t)\right] \bar{F}(t) d t}$.

Clearly, $\lim _{N \rightarrow \infty} C_{F}(T, N)=C(T)$ in (2) and $\lim _{T \rightarrow \infty} C_{F}(T, N)=C(N)$ in (5).

When $c_{T}=c_{N}$, differentiating $C_{F}(T, N)$ with respect to $T$ and setting it equal to zero,

$\int_{0}^{T}\left[1-G^{(N)}(t)\right] \bar{F}(t)[h(T)-h(t)] d t=\frac{c_{T}}{c_{F}-c_{T}}$.

The left-hand side increases with $T$ to $\infty$. Thus, there exists a unique $T_{F}^{*}\left(0<T_{F}^{*}<\infty\right)$ which satisfies (8), and

$$
C_{F}\left(T_{F}^{*}, N\right)=\left(c_{F}-c_{T}\right) h\left(T_{F}^{*}\right) .
$$

The left-hand side of (8) increases strictly with $N$ to that of (3), $T_{F}^{*}$ decreases strictly to $T^{*}$ in (3).

Forming the inequality $C_{F}(T, N+1)-C_{F}(T, N) \geq 0$,

$$
\int_{0}^{T}\left[1-G^{(N)}(t)\right] \bar{F}(t)[Q(T, N)-h(t)] \mathrm{dt} \geq \frac{c_{T}}{c_{F}-c_{T}} .
$$

Substituting (8) for (10),

$$
\mathrm{Q}(T, N) \geq h(T)
$$

which does not hold for any $N$, i.e., $N_{F}^{*}=\infty$. Thus, optimum policy to minimize $C_{F}(T, N)$ is $\left(T_{F}^{*}=T^{*}, N_{F}^{*}=\infty\right)$, where $T^{*}$ is given in (3). This shows that when the costs of preventive replacement are the equal, age replacement is more economical than the random policy.

Furthermore, if $Q(T, N)$ increases with $N$ to $h(T)$, then the left-hand side of (10) increases with $N$ to that of (3). Thus, if $T>T^{*}$, then there exists a unique minimum $N_{F}^{*}\left(1 \leq N_{F}^{*}<\infty\right)$ which satisfies (10), and decreases with $T$ to $N^{*}$ given in (6). Conversely, if $T \leq T^{*}$, then $N_{F}^{*}=\infty$. 
International Journal of Mathematical, Engineering and Management Sciences

Vol. 3, No. 2, 151-166, 2018

https://dx.doi.org/10.33889/IJMEMS.2018.3.2-012

\subsection{Replacement Last}

Suppose the unit is replaced at $T$ or at cycle $N$, whichever occurs last. Then, the expected cost rate is (Nakagawa, 2014)

$C_{L}(T, N)=\frac{c_{T}+\left(c_{F}-c_{T}\right)\left\{F(T)+\int_{T}^{\infty}\left[1-G^{(N)}(t)\right] d F(t)\right\}+\left(c_{N}-c_{T}\right) \int_{T}^{\infty} \bar{F}(t) d G^{(N)}(t)}{\int_{0}^{T} \bar{F}(t) d t+\int_{T}^{\infty}\left[1-G^{(N)}(t)\right] \bar{F}(t) d t}$.

Clearly, $\lim _{T \rightarrow 0} C_{L}(T, N)=\lim _{T \rightarrow \infty} C_{F}(T, N)$ and $\lim _{N \rightarrow 0} C_{L}(T, N)=\lim _{N \rightarrow \infty} C_{F}(T, N)$.

When $c_{T}=c_{N}$, differentiating $\mathrm{C}_{L}(T, N)$ with respect to $T$ and setting it equal to zero,

$\int_{0}^{T} \bar{F}(t)[h(T)-h(t)] d t-\int_{T}^{\infty}\left[1-G^{(N)}(t)\right] \bar{F}(t)[h(t)-h(T)] d t=\frac{c_{T}}{c_{F}-c_{T}}$.

The left-hand side of (12) increases with $T$ to $h(\infty)=\infty$. Thus, there exists a unique $T_{L}^{*}\left(0<T_{L}^{*}<\infty\right)$ which satisfies (12), and

$C_{L}\left(T_{L}^{*}, N\right)=\left(c_{F}-c_{T}\right) h\left(T_{L}^{*}\right)$

Letting $L_{F}(T)$ and $L_{L}(T)$ be the left-hand sides of (8) and (12),

$$
\begin{gathered}
L_{L F}(T)=L_{L}(T)-L_{F}(T) \\
=\int_{0}^{T} G^{(N)}(t) \bar{F}(t) t[h(T)-h(t)] d t-\int_{T}^{\infty}\left[1-G^{(N)}(t)\right] \bar{F}(t)[h(t)-h(T)] d t,
\end{gathered}
$$

which increases strictly with $T$ from $L_{L F}(0)<0$ to $\infty$. Thus, there exists a unique $T_{A}^{*}$ which satisfies $L_{L F}(T)=0$, and $T_{A}^{*}$ increases with $N$ to $\infty$. Thus, if $L_{F}\left(T_{A}^{*}\right) \geq c_{T} /\left(c_{F}-c_{T}\right)$, then $T_{F}^{*} \leq T_{L}^{*}$, and hence, from (9) and (13), replacement first is more economical than replacement last.

If $L_{F}\left(T_{A}^{*}\right)<c_{T} /\left(c_{F}-c_{T}\right)$, then $T_{L}^{*}<T_{F}^{*}$, and replacement last is more economical. This means that if the ratio of $c_{T} /\left(c_{F}-c_{T}\right)$ is large, we adopt replacement last, and if $N$ is large, replacement first is adopted.

\section{Replacement Overtime}

We suppose the unit is replaced preventively at the first completion of working cycles $Y_{j}(j=$ $1,2 \cdots)$ over $T$. We call this policy as replacement overtime, and the expected cost rate is (Nakagawa and Zhao, (2015).

$C_{O}(T)=\frac{c_{O}+\left(c_{F}-c_{O}\right) \sum_{j=0}^{\infty} \int_{0}^{T}\left[\int_{T-t}^{\infty} F(t+u) d G(u)\right] d G^{(j)}(t)}{\sum_{j=0}^{\infty} \int_{0}^{T}\left[\int_{0}^{\infty} \bar{G}(u) \bar{F}(t+u) d u\right] d G^{(j)}(t)}$,

where $c_{O}=$ replacement cost over $T$ with $c_{O}<c_{F}$. Optimum $T_{O}^{*}$ satisfies 
International Journal of Mathematical, Engineering and Management Sciences

Vol. 3, No. 2, 151-166, 2018

https://dx.doi.org/10.33889/IJMEMS.2018.3.2-012

$\widetilde{Q}(T) \sum_{j=0}^{\infty} \int_{0}^{T}\left[\int_{0}^{\infty} \bar{G}(u) \bar{F}(t+u) d u\right] d G^{(j)}(t)$

where

$$
-\sum_{j=0}^{\infty} \int_{0}^{T}\left[\int_{T-t}^{\infty} F(t+u) d G(u)\right] d G^{(j)}(t)=\frac{c_{O}}{c_{F}-c_{O}}
$$

$\tilde{Q}(T, N) \equiv \frac{\int_{T}^{\infty}\left[G^{(N)}(t)-G^{(N+1)}(t)\right] d F(t)}{\int_{T}^{\infty}\left[G^{(N)}(t)-G^{(N+1)}(t)\right] \bar{F}(t) d t}$,

and $\widetilde{Q}(T) \equiv \widetilde{Q}(T, 0)$.

When $G(t)=1-e^{-\theta t},(15)$ is

$\tilde{Q}_{1}(T) \int_{0}^{T} \bar{F}(t) d t-F(T)=\frac{c_{O}}{c_{F}-c_{O}}$

where

$\tilde{Q}_{1}(T) \equiv \frac{\int_{T}^{\infty} e^{-\theta t} d F(t)}{\int_{T}^{\infty} e^{-\theta t} \bar{F}(t) d t}$

increases with $T$ to $h(\infty)=\infty$. Thus, there exists a unique $T_{O}^{*}\left(0<T_{O}^{*}<\infty\right)$ which satisfies (16), and the resulting cost rate is

$c_{O}\left(T_{O}^{*}\right)=\left(c_{F}-c_{O}\right) \tilde{Q}_{1}\left(T_{O}^{*}\right)=\frac{c_{O}+\left(c_{F}-c_{O}\right) F\left(T_{O}^{*}\right)}{\int_{0}^{T_{O}^{*}} \bar{F}(t) d t}$

Noting that $\tilde{Q}_{1}(T)>h(T)$ for $0 \leq T<\infty, T_{O}^{*}<T^{*}$, and hence, from (2) and (17), $c_{O}\left(T_{O}^{*}\right)>$ $\mathrm{C}\left(T^{*}\right)$ when $c_{O}=c_{T}$, i.e., age replacement is more economical than the overtime policy.

\subsection{Replacement Overtime First}

Suppose the unit is replaced over $T(0 \leq T \leq \infty)$ or at cycle $N(N=1,2, \cdots)$, whichever occurs first. Then, the expected cost rate is (Nakagawa and Zhao, X. 2015).

$$
C_{O F}(T, N)=\frac{\begin{array}{c}
c_{O}+\left(c_{F}-c_{O}\right) \sum_{j=0}^{N-1} \int_{0}^{T}\left\{\int_{0}^{\infty}[F(t+u)-F(t)] d G(u)\right\} d G^{(j)}(t) \\
+\left(c_{N}-c_{O}\right) \int_{0}^{T} \bar{F}(t) d G^{(N)}(t)
\end{array}}{\sum_{j=0}^{N-1} \int_{0}^{T}\left[\int_{0}^{\infty} \bar{G}(u) \bar{F}(t+u) d u\right] d G^{(j)}(t)} .
$$

Clearly, $\lim _{T \rightarrow \infty} C_{O F}(T, N)=C(N)$ in $(5)$ and $\lim _{N \rightarrow \infty} C_{O F}(T, N)=C_{O}(T)$ in (14).

When $G(t)=1-e^{-\theta t}$ and $c_{O}=c_{N}$, optimum $T_{O F}^{*}$ satisfies

$\tilde{Q}_{1}(T) \sum_{j=0}^{N-1} \int_{0}^{T} \frac{(\theta t)^{j}}{j !} e^{-\theta t} \bar{F}(t) d t-\sum_{j=0}^{N-1} \int_{0}^{T} \frac{(\theta t)^{j}}{j !} e^{-\theta t} d F(t)=\frac{c_{O}}{c_{F}-c_{O}}$ 
International Journal of Mathematical, Engineering and Management Sciences

Vol. 3, No. 2, 151-166, 2018

https://dx.doi.org/10.33889/IJMEMS.2018.3.2-012

The left-hand side of (19) increases with $T$ to $h(\infty)$. Thus, there exists a unique $T_{O F}^{*}(0<$ $T_{O F}^{*}<\infty$ ) which satisfies (19), and the resulting cost rate is

$C_{O F}\left(T_{O F}^{*}, N\right)=\left(c_{F}-c_{O}\right) \widetilde{Q}_{1}\left(T_{O F}^{*}\right)$.

Noting that the left-hand side of (19) increases strictly with $N$ to that of (16), $T_{O F}^{*}$ decreases strictly with $N$ to $T_{O}^{*}$ given in (16).

Forming the inequality $C_{O F}(T, N+1)-C_{O F}(T, N) \geq 0$,

$$
\begin{gathered}
Q_{2}(T, N-1) \sum_{j=0}^{N-1}\left[\frac{(\theta T)^{j}}{j !} \int_{T}^{\infty} e^{-\theta t} \bar{F}(t) d t+\int_{0}^{T} \frac{(\theta t)^{j}}{j !} \bar{F}(t) d t\right] \\
-\sum_{j=0}^{N-1}\left[\frac{(\theta T)^{j}}{j !} \int_{T}^{\infty} e^{-\theta t} d F(t)+\int_{0}^{T} \frac{(\theta t)^{j}}{j !} e^{-\theta t} d F(t)\right] \geq \frac{c_{O}}{c_{F}-c_{O}}
\end{gathered}
$$

where

$Q_{2}(T, N) \equiv \frac{\int_{0}^{T}(\theta t)^{N}\left[\int_{t}^{\infty} e^{-\theta u} d F(u)\right] d t}{\int_{0}^{T}(\theta t)^{N}\left[\int_{t}^{\infty} e^{-\theta u} \bar{F}(u) d u\right] d t}<\tilde{Q}_{1}(T)$

increases strictly with $N$ to $\widetilde{\mathrm{Q}}_{1}(\mathrm{~T})$ and increases with $\mathrm{T}$ (Nakagawa, 2014). Substituting (19) for $(21)$,

$$
Q_{2}(T, N) \geq \tilde{Q}_{1}(T)
$$

which does not hold for any $N$, i.e., $N_{O F}^{*}=\infty$. Thus, optimum policy to minimize $C_{O F}(T, N)$ is $\left(T_{O F}^{*}=T_{O}^{*}, N_{O F}^{*}=\infty\right)$, where $T_{O}^{*}$ is given in (16). This shows that when the costs of preventive replacement are equal, replacement overtime is more economical than random policy.

Furthermore, noting that $Q_{2}(T, N)$ increase with $N$ to $\tilde{Q}_{1}(T)$ (Nakagawa and Zhao, 2015), the left-hand side of (21) increase strictly with $N$ to that of (16). Thus, if $T>T_{O}^{*}$, then there exists a unique minimum $N_{O F}^{*}\left(1 \leq N_{O F}^{*}<\infty\right)$ which satisfies (21), and decrease with $T$ to $N^{*}$ given in (6). Conversely, if $T \leq T_{O}^{*}$, then $N_{O F}^{*}=\infty$.

\subsection{Replacement Overtime Last}

Suppose the unit is replaced over time $T$ or at cycle $N$, whichever occurs last. Then, the expected cost rate is (Nakagawa and Zhao, 2015).

$c_{O}+\left(c_{N}-c_{O}\right) \int_{\mathrm{T}}^{\infty} \bar{F}(t) d G^{(N)}(t)+\left(c_{F}-c_{O}\right)\left(\int_{0}^{\infty} F(t) d G^{(N)}(t)\right.$
$C_{O L}(T, N)=\frac{\left.+\sum_{j=N}^{\infty} \int_{0}^{T}\left\{\int_{0}^{\infty}[F(t+u)-F(t)] d G(u)\right\} d G^{(j)}(t)\right)}{\int_{0}^{\infty}\left[1-G^{(N)}(t)\right] \bar{F}(t) d t+\sum_{j=N}^{\infty} \int_{0}^{T}\left[\int_{0}^{\infty} \bar{G}(u) \bar{F}(t+u) d u\right] d G^{(j)}(t)}$.

Clearly, $\lim _{T \rightarrow 0} C_{O L}(T, N)=\mathrm{C}(N)$ in (5) and $\lim _{N \rightarrow 0} C_{O L}(T, N)=C_{O}(T)$ in (14).

When $G(t)=1-e^{-\theta t}$ and $c_{O}=c_{N}$, optimum $T_{O L}^{*}$ satisfies 
International Journal of Mathematical, Engineering and Management Sciences

Vol. 3, No. 2, 151-166, 2018

https://dx.doi.org/10.33889/IJMEMS.2018.3.2-012

$\widetilde{Q}_{1}(T) \sum_{j=0}^{N-1} \int_{T}^{\infty} \frac{(\theta t)^{j}}{j !} e^{-\theta t} \bar{F}(t) d t-\sum_{j=0}^{N-1} \int_{T}^{\infty} \frac{(\theta t)^{j}}{j !} e^{-\theta t} d F(t)$

$+\tilde{Q}_{1}(T) \int_{0}^{T} \bar{F}(t) d t-F(T)=\frac{c_{O}}{c_{F}-c_{O}}$.

The left-hand side increase with $T$ to $h(\infty)=\infty$. Thus, there exists a unique $T_{O L}^{*}\left(0<T_{O L}^{*}<\right.$ $\infty)$ which satisfies (23), and

$C_{O L}\left(T_{O L}^{*}, \mathrm{~N}\right)=\left(c_{F}-c_{O}\right) \tilde{Q}_{1}\left(T_{O L}^{*}\right)$

Noting that the left-hand side of (23) increase with $N$ from that of (16), $T_{O L}^{*}$ decreases with $N$ from $T_{O}^{*}$ given in (16).

Forming the inequality $C_{O L}(T, N+1)-C_{O L}(T, N) \geq 0$,

$$
\begin{aligned}
& \tilde{Q}_{2}(T, N-1)\left[\int_{0}^{T} \bar{F}(t) d t+\sum_{j=0}^{N-1} \int_{T}^{\infty} \frac{(\theta t)^{j}}{j !} e^{-\theta t} \bar{F}(t) d t+\sum_{j=N}^{\infty} \frac{(\theta t)^{j}}{j !} \int_{T}^{\infty} e^{-\theta t} \bar{F}(t) d t\right]- \\
& \sum_{j=N}^{\infty}\left[\frac{(\theta t)^{j}}{j !} \int_{T}^{\infty} e^{-\theta t} d F(t)-\int_{T}^{\infty} \frac{(\theta t)^{j}}{j !} e^{-\theta t} d F(t)\right]-1 \geq \frac{c_{O}}{c_{F}-c_{O}}
\end{aligned}
$$

where

$\tilde{Q}_{2}(T, N) \equiv \frac{\int_{T}^{\infty}(\theta t)^{N}\left[\int_{t}^{\infty} e^{-\theta u} d F(u)\right] d t}{\int_{T}^{\infty}(\theta t)^{N}\left[\int_{t}^{\infty} e^{-\vartheta u} \bar{F}(t) d u\right] d t}<\tilde{Q}_{1}(T)$,

increases with $N$ from $\tilde{Q}_{1}(T)$ and increases with $T$ to $h(\infty)$. Substituting (23) for (25),

$$
\tilde{Q}_{2}(T, N-1) \geq \tilde{Q}_{1}(T),
$$

which always holds for any $T$, i.e., $N_{O L}^{*}=0$. Thus, optimum policy to minimize $C_{O L}(T, N)$ is $\left(T_{O L}^{*}=T_{O}^{*}, N_{O L}^{*}=\infty\right)$, where $T_{O}^{*}$ is given in (16). This shows that when the costs of preventive replacement are equal, replacement overtime is more economical than random policy.

Furthermore, noting that $\tilde{Q}_{2}(T, N)$ increases with $N$ from $\widetilde{Q}_{1}(T)$, the left-hand side of (25) increase with $N$ from

$$
\begin{gathered}
\tilde{Q}_{2}(T, 0)\left[\int_{0}^{T} \bar{F}(t) d t+\int_{T}^{\infty} e^{-\theta(t-T)} \bar{F}(t) d t\right]+\int_{T}^{\infty}\left[1-e^{-\theta(t-T)}\right] d F(t)-1 \\
\geq \tilde{Q}_{1}(T) \int_{0}^{T} \bar{F}(t) d t-F(T)
\end{gathered}
$$

which agrees with that of (16). Thus, if $T<T_{O}^{*}$, then there exists a unique and minimum $N_{O L}^{*}\left(1 \leq N_{O F}^{*}<\infty\right)$ which satisfies (25), and decreases with $T$ from $N^{*}$ given in (6). Conversely, if $T \geq T_{O}^{*}$, then $N_{O L}^{*}=0$. 
International Journal of Mathematical, Engineering and Management Sciences

Vol. 3, No. 2, 151-166, 2018

https://dx.doi.org/10.33889/IJMEMS.2018.3.2-012

\section{Replacement Middle}

Suppose the unit is replaced at $T, Y_{1}$ or $Y_{2}$, whichever occurs first, where each $Y_{i}(i=1,2)$ has distribution $G_{i}(t) \equiv \operatorname{Pr}\left\{Y_{i} \leq t\right\}$. Then, the expected cost rate is

$$
\begin{gathered}
c_{T}+\left(c_{1}-c_{T}\right) \int_{0}^{T} \bar{F}(t) \bar{G}_{2}(t) d G_{1}(t) \\
C_{2 F}(T)=\frac{+\left(c_{2}-c_{T}\right) \int_{0}^{T} \bar{F}(t) \bar{G}_{1}(t) d G_{2}(t)+\left(c_{F}-c_{T}\right) \int_{0}^{T} \bar{G}_{1}(t) \bar{G}_{2}(t) d F(t)}{\int_{0}^{T} \bar{F}(t) \bar{G}_{1}(t) \bar{G}_{2}(t) d t}
\end{gathered}
$$

where $c_{i}=$ replacement cost at $Y_{i}$ with $c_{i}<c_{F}(i=1,2)$ and $c_{T}$ and $c_{F}$ are given in (2).

When $c_{i}=c_{T}$, optimum $T_{2 F}^{*}$ to minimize $C_{2 F}(T)$ satisfies

$$
\int_{0}^{T} \bar{F}(t) \bar{G}_{1}(t) d G_{2}(t)[h(T)-h(t)] \mathrm{dt}=\frac{c_{T}}{c_{F}-c_{T}} .
$$

The left-hand side of (27) increases with $T$ from 0 to $h(\infty)=\infty$. Thus, there exists a unique $T_{2 F}^{*}\left(0<T_{2 F}^{*}<\infty\right)$ which satisfies $(27)$, and the resulting cost rate is

$C_{2 F}\left(T_{2 F}^{*}\right)=\left(c_{F}-c_{T}\right) h\left(T_{2 F}^{*}\right)$.

Next, suppose the unit is replaced at times $T, Y_{1}$ or $Y_{2}$, whichever occurs last. Then, the expected cost rate is

$$
C_{2 L}(T)=\frac{c_{T}+\left(c_{1}-c_{T}\right) \int_{T}^{\infty} \bar{F}(t) G_{2}(t) d G_{1}(t)+\left(c_{2}-c_{T}\right) \int_{T}^{\infty} \bar{F}(t) G_{1}(t) d G_{2}(t)}{+\left(c_{F}-c_{T}\right)\left[1-\int_{T}^{\infty} G_{1}(t) G_{2}(t) d F(t)\right]} .
$$

When $c_{i}=c_{T}$, optimum $T_{2 L}^{*}$ to minimize $C_{2 L}(T)$ satisfies

$$
\int_{0}^{T} \bar{F}(t)[h(T)-h(t)] d t-\int_{T}^{\infty}\left[1-G_{1}(t) G_{2}(t)\right] \bar{F}(T)[h(t)-h(T)] d t=\frac{c_{T}}{c_{F}-c_{T}} .
$$

The left-hand side of (30) increases strictly with $T$ to $h(\infty)=\infty$. Thus, there exists a finite and unique $T_{2 L}^{*}\left(0<T_{2 L}^{*}<\infty\right)$ which satisfies (30), and the resulting cost rate is

$$
C_{2 L}\left(T_{2 L}^{*}\right)=\left(c_{F}-c_{T}\right) h\left(T_{2 L}^{*}\right) \text {. }
$$

Finally, suppose the unit is replaced at times $T, Y_{1}$ or $Y_{2}$, whichever occurs middle. We call this policy as replacement middle (Zhao et al., 2015), and the expected cost rate is

$$
\begin{gathered}
c_{T}+\left(c_{1}-c_{T}\right)\left[\int_{0}^{T} \bar{F}(t) G_{2}(t) d G_{1}(t)+\int_{T}^{\infty} \bar{F}(t) \bar{G}_{2}(t) d G_{1}(t)\right] \\
+\left(c_{2}-c_{T}\right)\left[\int_{0}^{T} \bar{F}(t) G_{1}(t) d G_{2}(t)+\int_{T}^{\infty} \bar{F}(t) \bar{G}_{1}(t) d G_{2}(t)\right] \\
C_{2 M}(T)=\frac{+\left(c_{F}-c_{T}\right)\left\{\int_{0}^{T}\left[1-G_{1}(t) G_{2}(t)\right] d F(t)+\int_{T}^{\infty} \bar{G}_{1}(t) \bar{G}_{2}(t) d F(t)\right\}}{\int_{0}^{T}\left[1-G_{1}(t) G_{2}(t)\right] \bar{F}(t) d t+\int_{T}^{\infty} \bar{F}(t) \bar{G}_{1}(t) \bar{G}_{2}(t) d t} .
\end{gathered}
$$


International Journal of Mathematical, Engineering and Management Sciences

Vol. 3, No. 2, 151-166, 2018

https://dx.doi.org/10.33889/IJMEMS.2018.3.2-012

when $c_{i}=c_{T}$, optimum $T_{2 M}^{*}$ to minimize $C_{2 M}(T)$ satisfies

$$
\begin{aligned}
& \int_{0}^{T} \bar{F}(t)\left[1-G_{1}(t) G_{2}(t)\right][h(T)-h(t)] d t \\
& -\int_{T}^{\infty} \bar{F}(t) \bar{G}_{1}(t) \bar{G}_{2}(t)[h(t)-h(T)]=\frac{c_{T}}{c_{F}-c_{T}} .
\end{aligned}
$$

The left-hand side of (33) increases with $T$ to $\infty$. Thus, there exists a unique $T_{2 M}^{*}\left(0<T_{2 M}^{*}<\infty\right)$ which satisfies (33), and

$\mathrm{C}_{2 M}\left(T_{2 M}^{*}\right)=\left(c_{F}-c_{T}\right) h\left(T_{2 M}^{*}\right)$.

\subsection{Comparison of Three Polices}

Let $L_{F}(T), L_{L}(T)$ and $L_{M}(T)$ be the left-hand sides of (27), (30) and (33), respectively. From (27) and (30),

$$
\begin{aligned}
L_{L F}(T) \equiv & L_{L}(T)-L_{F}(T)=\int_{0}^{T}\left[1-\bar{G}_{1}(t) \bar{G}_{2}(t)\right][h(T)-h(t)] d t \\
& -\int_{T}^{\infty} \bar{F}(t)\left[1-G_{1}(t) G_{2}(t)\right][h(t)-h(T)] d t
\end{aligned}
$$

which increases with $T$ from $L_{L F}(0)<0$ to $\infty$. Thus, there exists a unique $\widetilde{\mathrm{T}}_{\mathrm{LF}}\left(0<\widetilde{\mathrm{T}}_{\mathrm{LF}}<\right.$ $\infty)$ which satisfies $L_{L F}(T)=0$.

From (27) and (33),

$$
\begin{aligned}
L_{M F}(T) \equiv L_{M}(T) & -L_{F}(T)=\int_{0}^{T}\left[G_{1}(t) \bar{G}_{2}(t)+\bar{G}_{1}(t) G_{2}(t)\right][h(T)-h(t)] d t \\
& -\int_{T}^{\infty} \bar{F}(t) \bar{G}_{1}(t) \bar{G}_{2}(t)[h(t)-h(T)] d t
\end{aligned}
$$

which increases strictly with $\mathrm{T}$ from $\mathrm{L}_{\mathrm{MF}}(0)<0$ to $\infty$. Thus, there exists unique $\widetilde{\mathrm{T}}_{\mathrm{MF}}(0<$ $\widetilde{\mathrm{T}}_{\mathrm{MF}}<\infty$ ) which satisfies $L_{M F}(T)=0$.

From (30) and (33)

$$
\begin{gathered}
L_{L M}(T) \equiv L_{L}(T)-L_{M}(\mathrm{~T})=\int_{0}^{T} \bar{F}(t) G_{1}(t) G_{2}(t)[h(T)-h(t)] d t \\
-\int_{T}^{\infty} \bar{F}(t)\left[G_{1}(t) \bar{G}_{2}(t)+\bar{G}_{1}(t) G_{2}(t)\right][h(t)-h(T)] d t
\end{gathered}
$$

which increases strictly with $T$ from $L_{L M}(0)<0$ to $\infty$. Thus, there exists a finite and unique $\widetilde{T}_{L M}\left(0<\tilde{T}_{L M}<\infty\right)$ which satisfies $L_{L M}(T)=0$. 
International Journal of Mathematical, Engineering and Management Sciences

Vol. 3, No. 2, 151-166, 2018

https://dx.doi.org/10.33889/IJMEMS.2018.3.2-012

The above discussions show the following comparative results:

(i) If $L_{M}\left(\tilde{T}_{M F}\right) \geq c_{T} /\left(c_{F}-c_{T}\right)$ and $L_{M}\left(\tilde{T}_{L M}\right) \geq c_{T} /\left(c_{F}-c_{T}\right)$, then the ranking is replacement first, middle, last.

(ii) If $L_{M}\left(\widetilde{T}_{M F}\right)<c_{T} /\left(c_{F}-c_{T}\right)<L_{M}\left(\tilde{T}_{L M}\right)$ and $L_{F}\left(\tilde{T}_{L F}\right) \geq c_{T} /\left(c_{F}-c_{T}\right)$, then the ranking is replacement middle, first, last.

(iii) If $L_{M}\left(\tilde{T}_{L M}\right)<c_{T} /\left(c_{F}-c_{T}\right)<L_{M}\left(\tilde{T}_{M F}\right)$ and $L_{F}\left(\widetilde{T}_{L F}\right)<c_{T} /\left(c_{F}-c_{T}\right)$, then the ranking is replacement middle, last, first.

(iv) If $L_{M}\left(\widetilde{T}_{M F}\right)<c_{T} /\left(c_{F}-c_{T}\right)$ and $L_{M}\left(\widetilde{T}_{L M}\right) \leq c_{T} /\left(c_{F}-c_{T}\right)$, then the ranking is replacement last, middle, first.

\section{General Age Replacement}

Suppose the unit is replaced at random times $Y_{i}$, where $Y_{i}(i=1,2, \ldots, n)$ are independent with each other and have general distributions $G_{i}(t)$ with finite means $1 / \theta_{i}$, respectively. Denoting $Y_{m} \equiv \min \left\{Y_{1}, Y_{2}, \ldots, Y_{n}\right\}$, it has a distribution

$\operatorname{Pr}\left\{Y_{m} \leq t\right\}=1-\prod_{i=1}^{n} \bar{G}_{i}(t)$

and denoting $Y_{M} \equiv \max \left\{Y_{1}, Y_{2}, \ldots, Y_{n}\right\}$, it has a distribution

$\operatorname{Pr}\left\{Y_{M} \leq t\right\}=\prod_{i=1}^{n} G_{i}(t)$

Note that (35) and (36) correspond to the respective failure distributions of a series system and a parallel system with $n$ units when $G_{i}(t)$ is the failure distribution of unit $i$ (Nakagawa, 2008), so that we call the above policies as redundant replacement.

Next, we consider the following four replacement policies:

(a) Replacement first: The unit is replaced at time $T$ or at time $Y_{m}$, whichever occurs first. Then, the unit is replaced at time $Y_{F} \equiv \min \left\{T, Y_{m}\right\}$ with a general distribution

$G_{F}(t) \equiv \operatorname{Pr}\left\{Y_{F} \leq t\right\}=\left\{\begin{array}{lrr} & 1-\prod_{i=1}^{n} \bar{G}_{i}(t) \quad t<T, & \\ 1 & & t \geq T .\end{array}\right.$

(b) Modified replacement first: The unit is replaced at time $T$ or at time $Y_{M}$, whichever occurs first. Then, the unit is replaced at time $\tilde{Y}_{F} \equiv \min \left\{T, Y_{M}\right\}$ with a general distribution

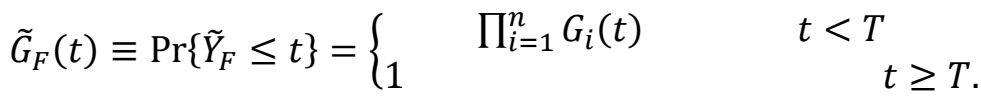

(c) Replacement last: The unit is replaced at time $T$ or at time $Y_{M}$, whichever occurs last. Then, the unit is replaced at time $Y_{L} \equiv \max \left\{T, Y_{M}\right\}$ with a general distribution 
International Journal of Mathematical, Engineering and Management Sciences

Vol. 3, No. 2, 151-166, 2018

https://dx.doi.org/10.33889/IJMEMS.2018.3.2-012

$G_{L}(t) \equiv \operatorname{Pr}\left\{\tilde{Y}_{F} \leq t\right\}= \begin{cases}0 & t<T, \\ \prod_{i=1}^{n} G_{i}(t) & t \geq T .\end{cases}$

(d) Modified replacement last: The unit is replaced at time $T$ or at time $Y_{m}$, whichever occurs last.

Then, the unit is replaced at time $\tilde{Y}_{L} \equiv \max \left\{T, Y_{m}\right\}$ with a general distribution

$\tilde{G}_{L}(t) \equiv \operatorname{Pr}\left\{Y_{L} \leq t\right\}= \begin{cases}0 & t<T \\ 1-\prod_{i=1}^{n} \bar{G}_{i}(t) & t \geq T .\end{cases}$

From the definitions of $Y_{i}$ and $\tilde{Y}_{i}(i=F, L), Y_{F} \leq \tilde{Y}_{F} \leq \tilde{Y}_{L} \leq Y_{L}$, and hence, $G_{F}(t) \geq \tilde{G}_{F}(t) \geq$ $\tilde{G}_{L}(t) \geq G_{L}(t)$.

\subsection{Replacement First}

Suppose the unit is replaced at time $Y_{F}$ in case (a). Then, replacing $G(t)$ with $G_{F}(t)$ in (37), the expected cost rate is (Chen et al., 2016)

$C_{G F}(T)=\frac{c_{R}+\left(c_{F}-c_{R}\right) \int_{0}^{T} \prod_{i=1}^{n} \bar{G}_{i}(t) d F(t)}{\int_{0}^{T} \bar{F}(t) \prod_{i=1}^{n} \bar{G}_{i}(t) d t}$,

where $c_{R}=$ replacement cost at time $Y_{i}$ with $c_{R}<c_{F}$.

Optimum $T_{F}^{*}$ to minimize $C_{G F}(T)$ satisfies

$\int_{0}^{T} \bar{F}(t)[h(T)-h(t)] \prod_{i=1}^{n} \bar{G}_{i}(t) d t=\frac{c_{R}}{c_{F}-c_{R}}$.

The left-hand side of (42) increases with $T$ to $h(\infty)=\infty$. Thus, there exists a unique $T_{F}^{*}(0<$ $T_{F}^{*}<\infty$ ) which satisfies (42), and the resulting cost rate is

$C_{G F}\left(T_{F}^{*}\right)=\left(c_{F}-c_{R}\right) h\left(T_{F}^{*}\right)$

Next, suppose the unit is replaced at time $\tilde{Y}_{F}$ in case (b). Then, replacing $G(t)$ with $G_{F}(t)$ in (38), the expected cost rate is (Chen et al., 2016)

$\tilde{C}_{G F}(T)=\frac{c_{R}+\left(c_{F}-c_{R}\right) \int_{0}^{T}\left[1-\prod_{i=1}^{n} G_{i}(t)\right] d F(t)}{\int_{0}^{T} \bar{F}(t)\left[1-\prod_{i=1}^{n} G_{i}(t)\right] d t}$.

Optimum $\tilde{T}_{F}^{*}$ to minimize $\tilde{C}_{G F}(T)$ satisfies

$\int_{0}^{T} \bar{F}(t)[h(T)-h(t)]\left[1-\prod_{i=1}^{n} G_{i}(t)\right] d t=\frac{c_{R}}{c_{F}-c_{R}}$.

The left-hand side of (45) increases with $T$ to $h(\infty)=\infty$. Thus, there exists a unique $\widetilde{T}_{F}^{*}(0<$ $\left.\tilde{T}_{F}^{*}<\infty\right)$ which satisfies (42), and the resulting cost rate is 
International Journal of Mathematical, Engineering and Management Sciences

Vol. 3, No. 2, 151-166, 2018

https://dx.doi.org/10.33889/IJMEMS.2018.3.2-012

$\tilde{C}_{G F}\left(\tilde{T}_{F}^{*}\right)=\left(c_{F}-c_{R}\right) h\left(\tilde{T}_{F}^{*}\right)$

Comparing (42) and (45)

$$
\int_{0}^{T} \bar{F}(t)[h(T)-h(t)]\left[1-\prod_{i=1}^{n} G_{i}(t)\right] d t-\int_{0}^{T} \bar{F}(t)[h(T)-h(t)] \prod_{i=1}^{n} \bar{G}_{i}(t) d t \geq 0
$$

which follows that $\tilde{T}_{F}^{*} \leq T_{F}^{*}$. Thus, from (43) and (46), $\tilde{C}_{G F}\left(\tilde{T}_{F}^{*}\right) \leq C_{G F}\left(T_{F}^{*}\right)$, i.e., modified replacement first is economical than replacement first.

\subsection{Replacement Last}

Suppose the unit is replaced at time $Y_{L}$ in case (c). Then, replacing $G(t)$ with $G_{L}(t)$ in (39), the expected cost rate is (Chen et al., 2016)

$C_{G L}(T)=\frac{c_{R}+\left(c_{F}-c_{R}\right)\left[1-\int_{T}^{\infty} \prod_{i=1}^{n} G_{i}(t) d F(t)\right]}{\int_{0}^{T} \bar{F}(t) d t+\int_{T}^{\infty} \bar{F}(t)\left[1-\prod_{i=1}^{n} G_{i}(t)\right] d t}$

Optimum $T_{L}^{*}$ to minimize $C_{G L}(T)$ satisfies

$\int_{0}^{T} \bar{F}(t)[h(T)-h(t)] d t-\int_{T}^{\infty} \bar{F}(t)[h(T)-h(t)]\left[1-\prod_{i=1}^{n} G_{i}(t)\right] d t=\frac{c_{R}}{c_{F}-c_{R}}$.

The left-hand side of (48) increases strictly with $T$ to $\infty$. Thus, there exists a unique $T_{L}^{*}(0<$ $T_{L}^{*}<\infty$ ) which satisfies (48), and the resulting cost rate is

$C_{G L}\left(T_{L}^{*}\right)=\left(c_{F}-c_{R}\right) \mathrm{h}\left(T_{L}^{*}\right)$.

Next, suppose the unit is replaced at time $\tilde{Y}_{L}$ in case (d). Then, replacing $G(t)$ with $\tilde{G}_{L}(t)$ in (39), the expected cost rate is (Chen et al., 2016)

$\tilde{C}_{G L}(T)=\frac{c_{R}+\left(c_{F}-c_{R}\right)\left\{1-\int_{T}^{\infty}\left[1-\prod_{i=1}^{n} \bar{G}_{i}(t)\right] d F(t)\right\}}{\int_{0}^{T} \bar{F}(t) d t+\int_{T}^{\infty} \bar{F}(t) \prod_{i=1}^{n} \bar{G}_{i}(t) d t}$.

Optimum $\tilde{T}_{L}^{*}$ to minimize $\tilde{C}_{G L}(T)$ satisfies

$\int_{0}^{T} \bar{F}(t)[h(T)-h(t)] d t-\int_{T}^{\infty} \bar{F}(t)[h(t)-h(T)] \prod_{i=1}^{n} \bar{G}_{i}(t) d t=\frac{c_{R}}{c_{F}-c_{R}}$.

The left-hand side of (51) increases with $T$ to $h(\infty)=\infty$. Thus, there exists a unique $\tilde{T}_{L}^{*}(0<$ $\left.\tilde{T}_{L}^{*}<\infty\right)$ which satisfies (51), and the resulting cost rate is

$\tilde{C}_{G L}\left(\tilde{T}_{L}^{*}\right)=\left(c_{F}-c_{R}\right) h\left(\tilde{T}_{L}^{*}\right)$.

Comparing (48) and (52), 
International Journal of Mathematical, Engineering and Management Sciences

Vol. 3, No. 2, 151-166, 2018

https://dx.doi.org/10.33889/IJMEMS.2018.3.2-012

$$
\int_{T}^{\infty} \bar{F}(t)[h(t)-h(T)]\left[1-\prod_{i=1}^{n} G_{i}(t)\right] d t-\int_{T}^{\infty} \bar{F}(t)[h(t)-h(T)] \prod_{i=1}^{n} \bar{G}_{i}(t) d t \geq 0
$$

which follows that $\tilde{T}_{L}^{*} \leq T_{L}^{*}$. Thus, from (49) and (52), $\tilde{C}_{G L}\left(\tilde{T}_{L}^{*}\right) \leq C_{G L}\left(T_{L}^{*}\right)$, i.e., modified replacement last is economical than replacement last.

Finally, it is assumed that $c_{R i}(i=1,2, \ldots, n)$ are respective replacement costs at times $Y_{i}$. Then, the expected cost rate in (41) becomes

$$
\begin{array}{r}
c_{F} \int_{0}^{T} \prod_{i=1}^{n} \bar{G}_{i}(t) d F(t)+c_{T} \bar{F}(T) \prod_{i=1}^{n} \bar{G}_{i}(t) \\
+\sum_{i=1}^{n} c_{R i} \int_{0}^{T} \bar{F}(t) \prod_{i=1, j \neq i}^{n} \bar{G}_{i}(t) d G_{i}(t)
\end{array} .
$$

Similarly, $\tilde{C}_{G F}(T)$ in (44), $C_{G L}(T)$ in (47) and $\tilde{C}_{G L}(T)$ in (50) can be obtained.

\section{Conclusions}

Age replacement model has been already challenged that the constant planned $T$ can be a random variable $Y$ to meet the random perforation need. In this paper, the so called replacement that is planned at time $T$ and random replacement planned at random time $Y$ have been introduced. Another random replacement that is planned at working cycles $N$ are also obtained. If we need to consider both age and random replacement policies with working cycles, we next have discussed replacement first, replacement last, replacement overtime and replacement middle. The motivations of these methods can be found in our literatures, so that we give the expected cost rates directly and discuss their optimum policies to minimize them analytically.

Another contribution of the paper is to give comparisons for these replacement policies. For examples, it has been shown that when the costs of preventive replacement are equal, age replacement is more economical than the random policy, age replacement is more economical than replacement overtime, replacement overtime is more economical than the random policy. Also, we have compared replacement first and replacement last analytically and showed that both have advantages in cost rates.

In order to formulate general replacement models of replacement first and last, we have obtained general distributions of replacement times, and the models of general replacement become our final target of the paper.

\section{Acknowledgement}

The authors thank the Grant-in-Aid for Scientific Research (C), Grant No. 15K03562 (2015-2017) from the Ministry of Education, Culture, Sports, Science in Japan and the Ministry of Science and Technology in Taiwan (No. MOST 104-2221-E-030-010). 
International Journal of Mathematical, Engineering and Management Sciences

Vol. 3, No. 2, 151-166, 2018

https://dx.doi.org/10.33889/IJMEMS.2018.3.2-012

\section{References}

Barlow, R. E., \& Proschan, F. (1996). Mathematical theory of reliability. Society for Industrial and Applied Mathematics.

Chen, M., Qian, C., Zhao, X., \& Nakagawa T. (2016). Replacement policy with a general model. Advanced Reliability and Maintenance Modeling VII (pp. 49-56). Magraw-Hill, Taiwan.

Karlin, S., \& Taylor, H. M. (1975). A first course in stochastic process. Academic Press, New York.

Nakagawa, T. (2005). Maintenance theory of reliability. Springer Science \& Business Media.

Nakagawa, T. (2008). Advanced reliability models and maintenance policies. Springer Science \& Business Media.

Nakagawa, T. (2014). Random maintenance policies. Springer, London.

Nakagawa, T., \& Zhao, X. (2015). Maintenance overtime policies in reliability theory: models with random working cycles. Springer.

Sarkar, A., Panja, S. C., \& Sarkar, B. (2011). Survey of maintenance policies for the last 50 years. International Journal of Software Engineering and Applications, 2(3), 130-148.

Zhao, X., \& Nakagawa, T. (2012). Optimization problems of replacement first or last in reliability theory. European Journal of Operational Research, 223(1), 141-149.

Zhao, X., Al-khalifa, K. N., Hamouda, A. M., \& Nakagawa, T. (2015). What is middle maintenance policy? Quality and Reliability Engineering International, 32(7), 2403-2414. 